Rev. Int. Contam. Ambie. 33 (1) 7-19, 2017

DOI: 10.20937/RICA.2017.33.01.01

\title{
EVALUACIÓN DE LA CONDICIÓN ECOLÓGICA DEL RÍO ZAHUAPAN
}

\author{
Irma MENA MEJÍA, Ángel BUSTAMANTE GONZÁLEZ*, Samuel VARGAS LÓPEZ, \\ José Isabel OLVERA HERNÁNDEZ y José Arturo MÉNDEZ ESPINOZA
}

Colegio de Postgraduados, Campus Puebla. Boulevard Forjadores de Puebla 205, Santiago Momoxpan, Municipio de San Pedro Cholula, Puebla, México, C.P. 72760

*Autor para correspondencia: angelb@colpos.mx

(Recibido abril 2015; aceptado junio 2016)

Palabras clave: bioindicadores, contaminación, macroinvertebrados, índice biótico

\section{RESUMEN}

Esta investigación tuvo como objetivo la evaluación integral de la condición ecológica del río Zahuapan con un enfoque de cuencas. Se tomó como base el protocolo de evaluación biótica en ecosistemas acuáticos, propuesto por la Agencia de Protección Ambiental de los Estados Unidos (EPA), el cual considera tres evaluaciones parciales: condición biótica, condición de hábitat y condición de calidad de agua. Para evaluar la condición del río, se ubicaron dos sitios de referencia en una zona de uso forestal y en nueve sitios perturbados, representativos de zonas de uso agrícola, urbano e industrial, a través de un sistema de información geográfica y recorridos de campo. La condición biótica se evaluó por medio de los macroinvertebrados presentes, identificados a nivel de familia. La condición de hábitat se evaluó considerando los parámetros establecidos por la EPA para ecosistemas acuáticos. La condición de calidad del agua se evaluó con un índice desarrollado a partir de mediciones de nueve parámetros físicos y químicos. La moda de la categoría de condición de las evaluaciones parciales fue considerada como la condición actual del río. Mientras que los sitios de referencia del río se ubicaron en una condición óptima, la condición del río en todos los sitios perturbados fue pobre, la peor condición en la escala de las evaluaciones.

Key words: bioindicators, pollution, macroinvertebrates, metrics

\begin{abstract}
This research aimed to assess the Zahuapan river health through a holistic and watershed approach. The Rapid Bioassessment Protocols for Use in Streams and Wadeable Rivers, of the US Environmental Protection Agency (EPA), was used as a support procedure to develop an integrated assessment, considering biotic, habitat and water quality partial assessments. In order to assess the river condition, two reference sites, located in a forest land, and nine perturbed sites, representatives of agricultural, urban and industrial lands, were selected in a geographical information system, with the support of field trips. The river biotic condition was based on families of benthic macroinvertebrates, the river habitat condition was assessed by using the habitat parameters established by the EPA, and the river water quality condition by developing an index from the nine measured physical and chemical parameters. The current river health
\end{abstract}


was considered as the mode of the category of the partial assessments. While the river health of the reference sites was optimum, it was poor for all the perturbed sites, the worst condition in the index scale.

\section{INTRODUCCIÓN}

El río Zahuapan es receptor de aguas residuales de origen urbano e industrial en el estado de Tlaxcala. Como consecuencia, la contaminación del río es notable (García-Nieto et al. 2011). Desde una perspectiva ecológica y de manejo de cuencas, la contaminación es solamente un aspecto del problema del deterioro del río. Se debe considerar también a la pérdida del hábitat ribereño y su biodiversidad, así como la del acuático, y la alteración del sistema hidrológico (caudales). Los conceptos de condición e integridad del río permiten trascender la visión de centrar la atención solamente en la contaminación. Este estudio tuvo como objetivo evaluar la condición e integridad del Río Zahuapan. Se tomó como base la condición de hábitat y parámetros físico - químicos de calidad del agua, mediante el protocolo de biomonitoreo rápido para ríos y corrientes basado en la presencia de macroinvertebrados bentónicos (Barbour et al. 1999).

En una evaluación integral del deterioro de un río son centrales los conceptos de cuenca y condición e integridad ecológica del río. La cuenca permite considerar la interacción de los componentes físicos, biológicos y socioeconómicos, así como tomar en cuenta relaciones espaciales de los procesos, desde los sitios de las partes altas a las partes bajas (Frissell et al. 1986). La integridad ecológica de los ecosistemas acuáticos se define en relación con una condición de referencia, donde los impactos de las actividades humanas son mínimos o imperceptibles. A partir de ahí se evalúa el grado en el cual los componentes físicos, químicos y biológicos de un ecosistema y sus relaciones están presentes, funcionan y se mantienen (Schallenberg et al. 2011). Integridad ecológica, salud del río y condición ecológica son términos que se consideran a veces como equivalentes en estudios de monitoreo de los ríos con el propósito de medir una condición total de los ecosistemas acuáticos, aunque para algunos esto es controversial (Oliveira y Cortes 2006).

El biomonitoreo o monitoreo biológico utiliza organismos bioindicadores o sus respuestas para determinar la condición o cambio del ambiente o de un ecosistema. A través de él se evalúan los efectos de la influencia humana sobre un ecosistema acuático (Karr 2006). Un bioindicador es un organismo (o parte de un organismo) que contiene información de la calidad del ambiente (Li et al. 2010). Para la evaluación de ríos, los macroinvertebrados bentónicos, las algas y los peces son los más utilizados.

Los métodos de biomonitoreo incluyen el uso de índices de diversidad, índices bióticos, métodos multimétricos, métodos multivariados y enfoques funcionales (Richards y Horn 2005, Li et al. 2010). El índice de integridad biótica (IBI, por sus siglas en inglés) ha sido ampliamente utilizado para evaluar la integridad o condición de los ríos (Harrison y Whitfield 2006). Barbour et al. (1999), desarrollaron un protocolo de biomonitoreo rápido para usarse en ríos y corrientes, basado en la presencia de perifiton, macroinvertebrados bentónicos y peces. Es un método multimétrico que evalúa también la calidad de la estructura del hábitat e incluye aspectos físcos y químicos del agua del río.

En México, los primeros estudios sobre bioindicadores de ríos se realizaron en los años 90. Lyons et al. (1995) desarrollaron un índice de integridad biótica para la región centro-noroeste de México, basado en comunidades de peces, a partir de 10 parámetros considerados en índices y que han sido utilizadas en regiones de Norteamérica. Ellos evaluaron también la calidad ambiental de los sitios a partir de variables del hábitat y de la calidad de agua, estandarizando los datos a una escala de 10 puntos y considerando el valor más bajo obtenido como indicador de la calidad ambiental menor. Para la misma región, Weigel et al. (2002) utilizaron ocho medidas para desarrollar un índice de integridad biótica basado en macroinvertebrados. Para la condición ambiental, evaluaron la perturbación como mínima, moderada y severa. Otros estudios orientaron el monitoreo a la evaluación de índices de diversidad (Godínez-Domínguez y González-Sansón 1999). Los índices bióticos utilizados inicialmente en México identificaron taxonómicamente a los macroinvertebrados hasta el nivel de género, aunque recientemente se ha considerado la identificación a nivel de familia como una opción, por la facilidad de identificación (Gamboa et al. 2008). 


\section{MATERIALES Y MÉTODOS}

\section{Área de estudio}

El estudio se realizó en la subcuenca del río Zahuapan(Fig. 1), la cual tiene una superficie de $1632.7 \mathrm{~km}^{2}$. El clima predominante es templado subhúmedo y solamente en las áreas con mayor altitud se presenta un clima semifrío subhúmedo. En la zona de estudio los tipos de vegetación que predominan son el pastizal, el bosque de táscate, el bosque de pino y el bosque de encino. En la subcuenca está asentada el $61.18 \%$ de la población del estado de Tlaxcala (INEGI 2010) y se tiene la presencia de una zona industrial importante.

\section{Identificación de factores de perturbación}

Las zonas de agricultura de riego, consideradas como fuentes no puntuales de contaminación, se delimitaron con la cartografía digital de uso de suelo vegetación, serie IV del Instituto Nacional de Estadística y Geografía. Se precisó la delimitación de uso de suelo con fotografías aéreas y se realizaron recorridos de campo para validarla. Los sitios de descarga de aguas residuales (fuentes puntuales de contaminantes) se ubicaron con el Localizador del Registro Público de Derechos de Agua (LOCREPDA), un sistema de información de la Comisión Nacional del Agua (CONAGUA 2012). Adicionalmente, a través de un sistema de información geográfica se relacionaron los sitios de descarga con el origen de las mismas: industrial, público-urbano, servicios y pecuario.

\section{Sitios de muestreo}

Para evaluar la condición del río se seleccionaron once sitios de muestreo (Fig. 1, S1 a S11), dos de ellos fueron sitios de referencia, localizados en ecosistemas forestales (S1 y S2), donde la perturbación por actividades humanas es mínima. Los demás sitios se ubicaron en áreas con influencia de alguno de los factores de perturbación de la condición del río: agrícola-pastizal (S3), agrícola (S4 y S11), urbano (S5, S9 y S10) e industrial y urbano-industrial (S6, S7 y S8). En todos los sitios se evaluaron en la primer quincena del mes de mayo de 2013 tres componentes: a) macroinvertebrados presentes, b) condición de hábitat y c) parámetros de calidad del agua. Se seleccionó un periodo de muestreo previo al establecimiento del periodo lluvioso para evitar alteraciones del caudal y de la presencia de macroinvertebrados.

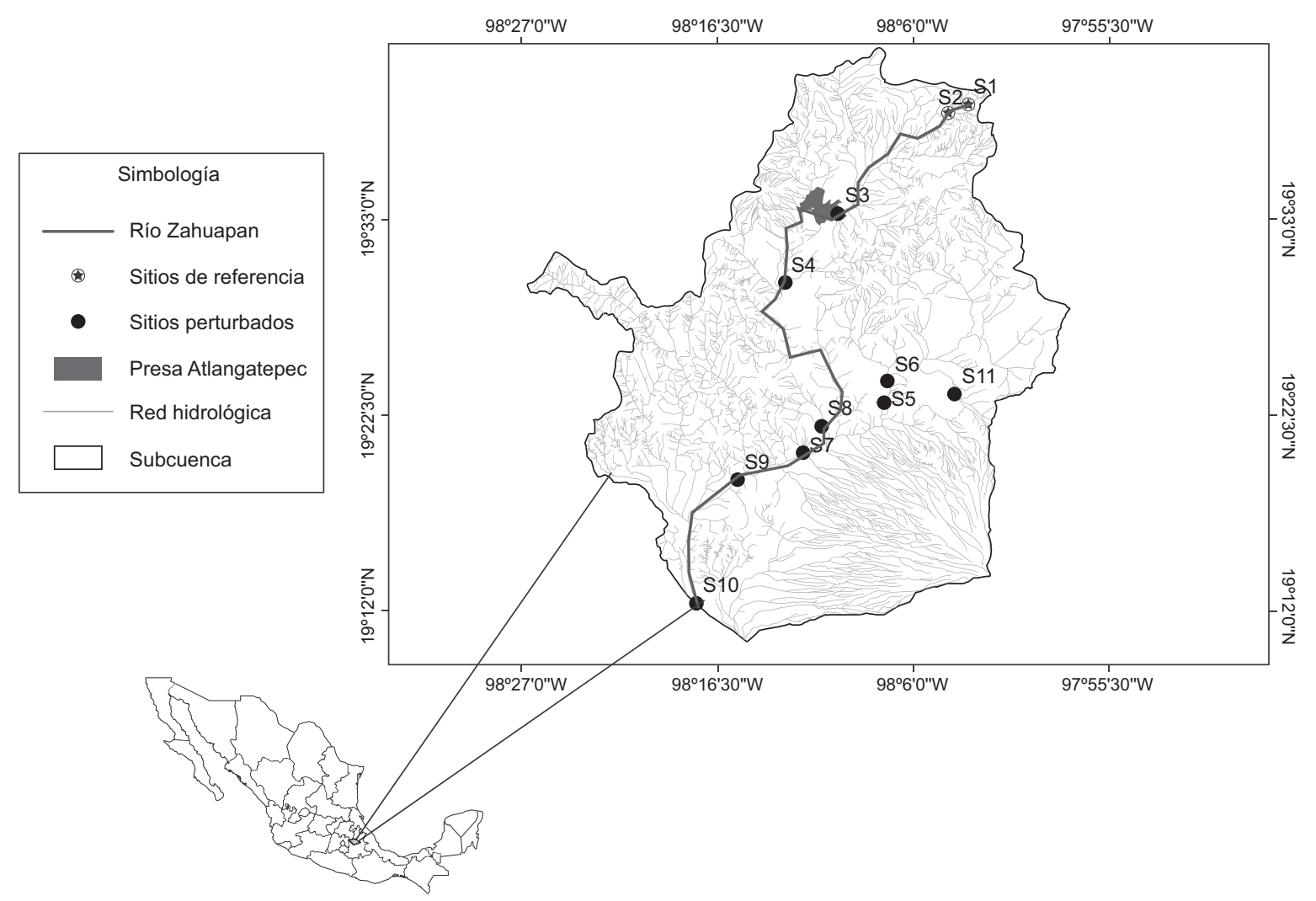

Fig. 1. Subcuenca del río Zahuapan 


\section{Muestreo de macroinvertebrados y condición biótica}

Se colectaron los macroinvertebrados en tramos de $100 \mathrm{~m}$ del río, con una red de marco rectangular de $0.5 \times 0.3 \mathrm{~m}$ y apertura de malla de $500 \mu \mathrm{m}$. En cada sitio se realizaron 20 golpes con la red sobre el lecho del río para dispersar y colectar a los organismos, de forma proporcional al tipo de hábitat, de acuerdo con el método propuesto por Barbour et al. (1999). Por ejemplo, si en un sitio el $50 \%$ del hábitat era de cantos rodados, entonces se daban 10 golpes con la red y el resto de los golpes se distribuían en los hábitats restantes (tocones, bancos con vegetación, macrófitas sumergidas y arena). Los organismos se colocaron en una charola de plástico marcada con cuadrículas de $6 \times 6 \mathrm{~cm}$, numeradas del 1 al 36. Con una tabla de números aleatorios se seleccionó una cuadrícula para colectar los organismos presentes repitiéndose el procedimiento hasta obtener 250 organismos. Los organismos se identificaron hasta el nivel de familia, utilizando un microscopio estereoscópico y las claves de identificación de Bouchard (2004), Oscoz (2009), Serrano y Zepeda (2010) y Gutiérrez-Fonseca (2010).

Se determinó la tolerancia de los organismos identificados a la perturbación de la calidad del agua del río y se identificó el grupo funcional de alimentación al que pertenecen (raspadores, depredadores, colectores, filtradores o fragmentadores), de acuerdo con los cuadros reportados por Bouchard (2004). Con las familias identificadas y el número de individuos por familia se calcularon los valores para cada sitio: número total de familias; número de familias de los órdenes Ephemeroptera, Plecopera, Trichoptera; porcentaje compuesto de Ephemeroptera, Plecoptera, Trichoptera (\% de EPT); porcentaje de Ephemeroptera; porcentaje de organismos tolerantes; Índice Biótico de Hilsenhoff (IBH); porcentaje de la familia presente más dominante ( $\%$ de familia dominante); porcentaje de raspadores; porcentaje de depredadores; porcentaje de colectores; porcentaje de filtradores y porcentaje de fragmentadores. El IBH en cada sitio se calculó con la fórmula: $\mathrm{IBH}=\Sigma \mathrm{XiTi} / \mathrm{n}$, donde Xi es el número de ejemplares de cada familia; Ti corresponde al valor de tolerancia para dicha familia y $n$ es el número total de organismos recolectados en la muestra.

Un criterio de selección de medidas para evaluar la condición biótica fue el análisis de correlación de Spearman entre las medidas calculadas. Un segundo criterio fue la relación de medias entre las medidas de los sitios perturbados y los sitios de referencia. Como tercer criterio se graficaron los índices bióticos con los parámetros de perturbación: conductividad eléctrica, cubrimiento de cantos rodados por sedimentos finos y ancho de la zona riparia (Herbst y Silldorf 2009). Se utilizó el cuadro I (Plafkin et al. 1989) para obtener un puntaje de los índices bióticos. Para el cálculo se utilizó el sitio S1 como referencia.

Se obtuvo la suma de puntaje de todos los índices bióticos para cada sitio. Se calculó el porcentaje que representó el puntaje de los sitios perturbados en relación con el puntaje del sitio S1. El valor obtenido (\% de referencia) se comparó con los valores del cuadro II para determinar la condición biótica del sitio.

CUADRO I. CRITERIOS DE PUNTAJE PARA LA CARACTERIZACIÓN DE LA CONDICIÓN BIÓTICA

\begin{tabular}{lcccc}
\hline \multirow{2}{*}{ Índice Biótico } & \multicolumn{3}{c}{$\begin{array}{c}\text { Criterios de puntaje de la } \\
\text { condición biótica }\end{array}$} \\
\cline { 2 - 5 } & 6 & 3 & 0 \\
\hline Número total de familias (a) & $>80 \%$ & 80 & $-40 \%$ & $<40 \%$ \\
IBH (b) & $>85 \%$ & 85 & $-50 \%$ & $<50 \%$ \\
Índice EPT (a) & $>90 \%$ & 90 & $-70 \%$ & $<70 \%$ \\
Porcentaje de la familia dominante (d) & $<30 \%$ & $50-30 \%$ & $<30 \%$ \\
Índice de pérdida de familias (e) & $<0.5 \%$ & $0.5-4 \%$ & $>4 \%$ \\
Relación raspadores/filtradores colectores (a, c) & $>50 \%$ & 25 & $-50 \%$ & $<25 \%$ \\
Relación EPT y abundancia de Chironomidae (a) & $>75 \%$ & 25 & $-75 \%$ & $<25 \%$ \\
$\%$ fragmentadores (a) & $>50 \%$ & 50 & $-25 \%$ & $<25 \%$ \\
\hline
\end{tabular}

(a) valor del sitio impactado/valor de referencia x 100, (b) valor del sitio de referencia/ valor del sitio impactado $\times 100$, (c) la determinación del grupo funcional de alimentación es independiente de la agrupación taxonómica, (d) porcentaje estimado para cada sitio perturbado, sin comparar con el de referencia, se ubica el valor del índice biótico en los rangos del cuadro. EPT = compuesto de Ephemeroptera, Plecoptera, Trichoptera.

Fuente: Plafkin et al. (1989) 
CUADRO II. CONDICIÓN BIÓTICA DE LOS SITIOS

\begin{tabular}{lll}
\hline$\%$ de referencia & Condición biótica & Características \\
\hline$>79 \%$ & $\begin{array}{l}\text { Óptima } \\
\text { (no impactada) }\end{array}$ & $\begin{array}{l}\text { Comparable con los } \\
\text { sitios de referencia, } \\
\text { composición trófica } \\
\text { balanceada, y una co- } \\
\text { munidad con domi- } \\
\text { nancia y composición } \\
\text { adecuada para el ta- } \\
\text { maño de la corriente, } \\
\end{array}$ \\
& $\begin{array}{l}\text { con un hábitat óptimo. } \\
\text { Subóptima } \\
\text { (moderadamente } \\
\text { impactada) }\end{array}$ & $\begin{array}{l}\text { Pérdida de familias } \\
\text { intolerantes reducción } \\
\text { del índice EPT. }\end{array}$ \\
\hline $72-29 \%$ & $\begin{array}{l}\text { Solo familias toleran- } \\
\text { tes están presentes (1 } \\
\text { (severamente }\end{array}$ & o 2 generalmente) \\
\hline$<21 \%$ & impactada) & Plecopter Trichoptera.
\end{tabular}

EPT $=$ compuesto de Ephemeroptera, Plecoptera, Trichoptera. El índice EPT es la relación \% de EPT en el sitio impactado / \% de EPT en el sitio de referencia.

Fuente: Plafkin et al. (1989).

\section{Parámetros de hábitat y condición de hábitat}

Respecto a la condición de hábitat, para cada parámetro listado en el cuadro III se asignó, a través de una evaluación visual, un valor de condición de hábitat de acuerdo con los lineamientos descritos por Barbour et al. (1999). Los rangos de condición para cada parámetro se describen en el cuadro III. Para cada sitio se sumaron los valores de todos los parámetros y así se obtuvo una calificación agregada.
Esta calificación se dividió entre 200, que es el valor máximo posible y se utilizaron los rangos del Departamento de Recursos Naturales de Vermont (VANR 2004) para evaluar la condición de hábitat de cada sitio. Estos rangos son: óptima $(0.85-1.0)$, subóptima $(0.65-0.84)$, marginal $(0.35-0.64)$ y pobre $(0.0-0.34)$.

Se realizó un análisis de conglomerados, utilizando el programa SPSS (Statistical Package for Social Sciences) versión 17, con los valores de condición de hábitat para identificar la agrupación de los sitios y observar si los sitios de referencia se separan de los sitios perturbados. Con base en el dendograma obtenido se identificaron grupos con diferente condición de hábitat.

\section{Medición de parámetros físicos y químicos}

Se determinaron los parámetros de $\mathrm{pH}$, total de sólidos disueltos, fósforo total, temperatura del agua, oxígeno disuelto, demanda biológica de oxígeno y amonio. La temperatura del agua y el oxígeno disuelto se midieron en los sitios de muestreo con un oxímetro marca Hanna modelo HI 8043. En laboratorio se determinaron el $\mathrm{pH}$ y el total de sólidos disueltos con un multiparámetro de calidad del agua marca Hach modelo Sension 156, el fósforo total por el método de PhosVer ${ }^{\circledR} 3$ con digestión de persulfato ácido, el amonio por el método de salicilato y la demanda biológica de oxígeno $\left(\mathrm{DBO}_{5}\right)$ por el método respirométrico de acuerdo con el procedimiento Hach GGA (glucosa/ácido glutámico), equipo BODTrack II (HACH 2003).

CUADRO III. VALORES DE LOS PARÁMETROS DE LA CONDICIÓN DE HÁBITAT DE LOS SITIOS

\begin{tabular}{llcccc}
\hline Parámetro & & \multicolumn{3}{c}{ Condición } \\
\cline { 3 - 5 } & & Óptima & Subóptima & Marginal & Pobre \\
\hline Alteración del cauce & & $20-16$ & $15-11$ & $10-6$ & $5-0$ \\
Estado del cauce & & $20-16$ & $15-11$ & $10-6$ & $5-0$ \\
Cubrimiento de cantos por sedimentos & & $20-16$ & $15-11$ & $10-6$ & $5-0$ \\
Estabilidad del banco & Derecho & $10-9$ & $8-6$ & $5-3$ & $2-0$ \\
& Izquierdo & $10-9$ & $8-6$ & $5-3$ & $2-0$ \\
Regímenes de velocidad y profundidad & & $20-16$ & $15-11$ & $10-6$ & $5-0$ \\
Sustrato para la epifauna & & $20-16$ & $15-11$ & $10-6$ & $5-0$ \\
Frecuencia de rápidos & & $20-16$ & $15-11$ & $10-6$ & $5-0$ \\
Ancho de la zona ribereña del banco & Derecho & $10-9$ & $8-6$ & $5-3$ & $2-0$ \\
& Izquierdo & $10-9$ & $8-6$ & $5-3$ & $2-0$ \\
Depósito de sedimento & & $20-16$ & $15-11$ & $10-6$ & $5-0$ \\
Protección vegetal del banco & Derecho & $10-9$ & $8-6$ & $5-3$ & $2-0$ \\
Total & Izquierdo & $10-9$ & $8-6$ & $5-3$ & $2-0$ \\
\hline
\end{tabular}

Fuente: Barbour et al. (1999) 
Se calculó el índice de calidad del agua (ICA) propuesto por el Consejo Ambiental de Ministros de Canadá (CCME 2001):

$I C A=100-\left[\sqrt[2]{F_{1}^{2}+F_{2}^{2}+F_{3}^{2}} / 1.732\right]$

El valor de 1.732 normaliza los valores resultantes a un rango de 0 a 100, donde 0 representa la peor condición de calidad de agua y 100 la mejor condición. F1 es el porcentaje de parámetros que no cumplen con un valor de referencia (número de parámetros que no cumplen con el valor de referencia / número total de variables $\times 100$ ). F2 es el porcentaje de valores individuales de los parámetros que no cumplen con el valor de referencia (número de valores de los parámetros que no cumplen con el valor de referencia / total de valores de los parámetros medidos $\times 100$ ). F3 es la amplitud con la que no se cumplió con el valor de referencia, calculada como una función asintótica que escala la suma normalizada de desviaciones (sne) para dar un rango de 0 a 100, mediante la siguiente ecuación: $F 3=[$ sne $/(0.01$ sne +0.01$)]$. Sne se estima como: sne $=\left[\sum \mathrm{e}_{\mathrm{i}}\right] /$ número de pruebas.

Una desviación $\left(\mathrm{e}_{\mathrm{i}}\right)$ es el número de veces que el valor individual de un parámetro fue mayor (o menor, si el valor de referencia es mínimo) que el máximo (mínimo) permisible, de acuerdo con el objetivo establecido (la protección de la vida acuática). Para el primer caso $\mathrm{e}_{=}$(Valor del parámetro i que no cumple con el valor de referencia / valor de referencia j para el parámetro i $)-1$. Para el segundo caso e $=($ valor de referencia j para el parámetro i / valor del parámetro i que no cumple con el valor de referencia) -1 .

Debido a que en México no existen valores de referencia para los parámetros físicos y químicos de calidad de agua en ríos, el fósforo total, la demanda biológica de oxígeno $\left(\mathrm{DBO}_{5}\right)$ y la temperatura se compararon con los valores de la NOM-001-SEMARNAT-1996, la cual establece los límites máximos permisibles de descargas de aguas residuales en aguas y bienes nacionales. Para el oxígeno disuelto, amonio y $\mathrm{pH}$ se consideraron valores de la Norma Canadiense para la Protección de Vida Acuática (CCME 2001, CCME 2007). El valor de referencia del total de sólidos disueltos en ríos se basó en los valores establecidos por el Departamento de Recursos Naturales del Estado de Iowa (IDNR 2009). La asignación de la condición del río se realizó conforme a los rangos de la Norma Canadiense para la Protección de Vida Acuática (CCME 2001), descritos en el cuadro IV.

\section{Evaluación de la condición global del río}

Se tabularon las categorías de calidad de los sitios
CUADRO IV. ÍNDICE Y CONDICIÓN DE CALIDAD DEL AGUA

\begin{tabular}{lcl}
\hline $\begin{array}{l}\text { Condición de } \\
\text { calidad del agua }\end{array}$ & Valor del índice & Características \\
\hline Excelente & $95-100$ & $\begin{array}{l}\text { Calidad del agua sin dete- } \\
\text { rioro o perturbación simi- } \\
\text { lar a sitios prístinos. }\end{array}$ \\
\hline Buena & $94-80$ & $\begin{array}{l}\text { Calidad del agua con me- } \\
\text { nor grado de deterioro con } \\
\text { respecto a la excelente, } \\
\text { en pocas ocasiones pue- } \\
\text { den no ser similares a las } \\
\text { naturales. }\end{array}$ \\
\hline Favorable & $79-65$ & $\begin{array}{l}\text { Calidad del agua con per- } \\
\text { turbación ocasional. }\end{array}$ \\
\hline Marginal & $64-45$ & $\begin{array}{l}\text { Calidad del agua con per- } \\
\text { turbación frecuente. }\end{array}$ \\
\hline Pobre & $44-0$ & $\begin{array}{l}\text { Calidad del agua con per- } \\
\text { turbación permanente. }\end{array}$ \\
\hline
\end{tabular}

obtenidos por las evaluaciones parciales de condición biótica, de hábitat y de calidad de agua. Se tomó la moda de la condición de las tres categorías como la condición representativa del sitio.

\section{RESULTADOS Y DISCUSIÓN}

\section{Factores de perturbación de la integridad eco- lógica}

Las fuentes puntuales de contaminación identificadas en la subcuenca son sitios de descarga de aguas residuales de origen industrial, público-urbano, servicios y pecuario. Los que tienen mayor incidencia sobre el río, tanto por su distribución como por los volúmenes de agua residual descargada, son los desarrollos industriales y los centros urbanos (Fig. 2, Cuadro V).

La agricultura de riego, como fuente de contaminación no puntual, ocupa una superficie pequeña en la subcuenca (Fig. 3). Se presenta en la parte norte, aguas abajo de la presa Atlangatepec (19 33'33" Latitud Norte y $98^{\circ} 11^{\prime} 10^{\prime \prime}$ Longitud Oeste), con una superficie de 374.8 ha y en la parte sur, en la salida de la subcuenca, con una superficie de 9969.0 ha.

\section{Condición biótica}

Se identificaron trece familias de macroinvertebrados (Cuadro VI). Los sitios de referencia tuvieron cuatro familias con tolerancia baja a la contaminación del agua y tres familias con tolerancia moderada. Dos 


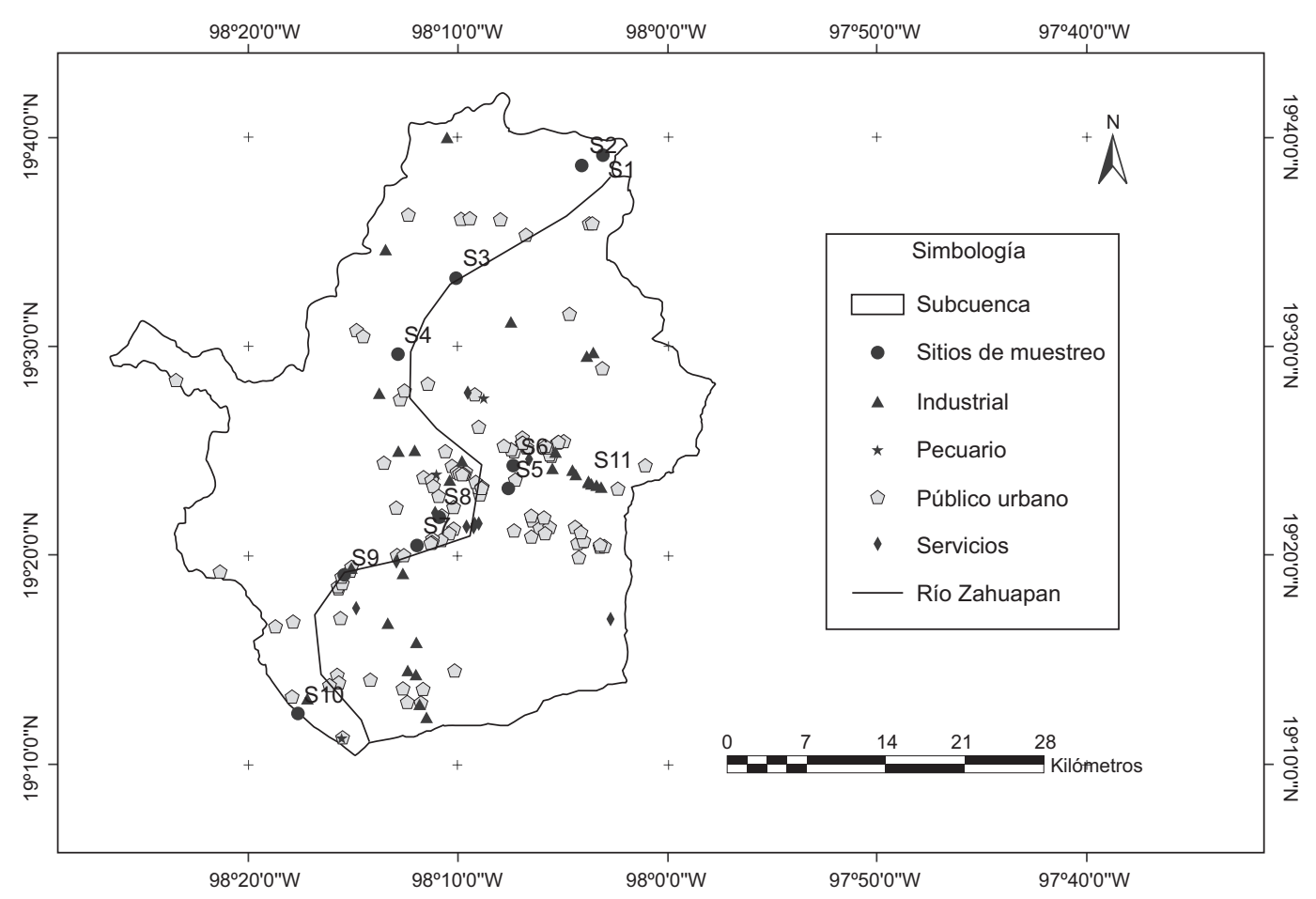

Fig. 2. Ubicación de los sitios de descargas de aguas residuales en la subcuenca del río Zahuapan

CUADRO V. VOLUMEN DE DESCARGAS EN LA RED HIDROLÓGICA DE LA SUBCUENCA DEL RÍO ZAHUAPAN

\begin{tabular}{lc}
\hline Tipo de descarga & Volumen de descarga $(\mathrm{L} / \text { día })^{*}$ \\
\hline Público-urbano & 40613497.0 \\
Industrial & 2818514.3 \\
Pecuario & 5840.0 \\
Servicios & 92805.7 \\
Total & 43530657.0 \\
\hline
\end{tabular}

* Fuente: CONAGUA (2012)

familias de tolerancia alta estuvieron presentes con muy pocos organismos. En los sitios perturbados se registró el mayor número de familias tolerantes a la contaminación. En los sitios S3 y S9 hubo presencia de oligoquetos, lo que coincide con lo reportado para zonas urbanas con contaminación por descargas de aguas residuales (Moreno y Callisto 2006, CorreaAraneda et al. 2010). En los sitios donde se combinan descargas industriales y urbanas (sitios S3, S5 y S9), sólo los organismos tolerantes están presentes, como la familia de Tubificidae y Glossiphoniidae. La presencia de organismos de la familia Tubificidade en los sitios S3 y S9 (el primero se ubica aguas abajo de la presa de Atlangatepec y el segundo en una zona de confluencia de agricultura de riego y descargas urbanas), indica altas concentraciones de materia orgánica, ya que organismos de esta familia se relacionan con ello (Martins et al. 2008). En los sitios S6, S7, S8, S10 y S11 la descarga industrial eliminó a los organismos totalmente, lo que apunta hacia un alto nivel de contaminación del agua, asociado con las descargas de aguas residuales (industrial, público-urbano, servicios y pecuario), la presencia de zonas agrícolas y la fuerte alteración del hábitat por modificación del cauce del río.

El análisis de correlación de Spearman mostró que el porcentaje de EPT, el porcentaje de organismos tolerantes, el porcentaje de fragmentadores y el número de familias EPT tuvieron el menor número de correlaciones altas $(r>0.8)$ respecto a otros índices bióticos. El análisis de relación de medias entre los sitios de referencia y los perturbados indicó que los índices bióticos sensibles a la perturbación son: el porcentaje del número total de familias, el porcentaje de colectores, el porcentaje de depredadores, el porcentaje de organismos tolerantes, el porcentaje de la familia dominante y el IBH. Las gráficas que relacionan índices bióticos con los parámetros de perturbación (conductividad, cubrimiento de cantos rodados por sedimentos finos y ancho de la zona ribereña), indicaron que el número total de familias, el porcentaje de organismos tolerantes, el IBH, el porcentaje de familia dominante y el porcentaje de colectores se relacionan con los parámetros analizados. 


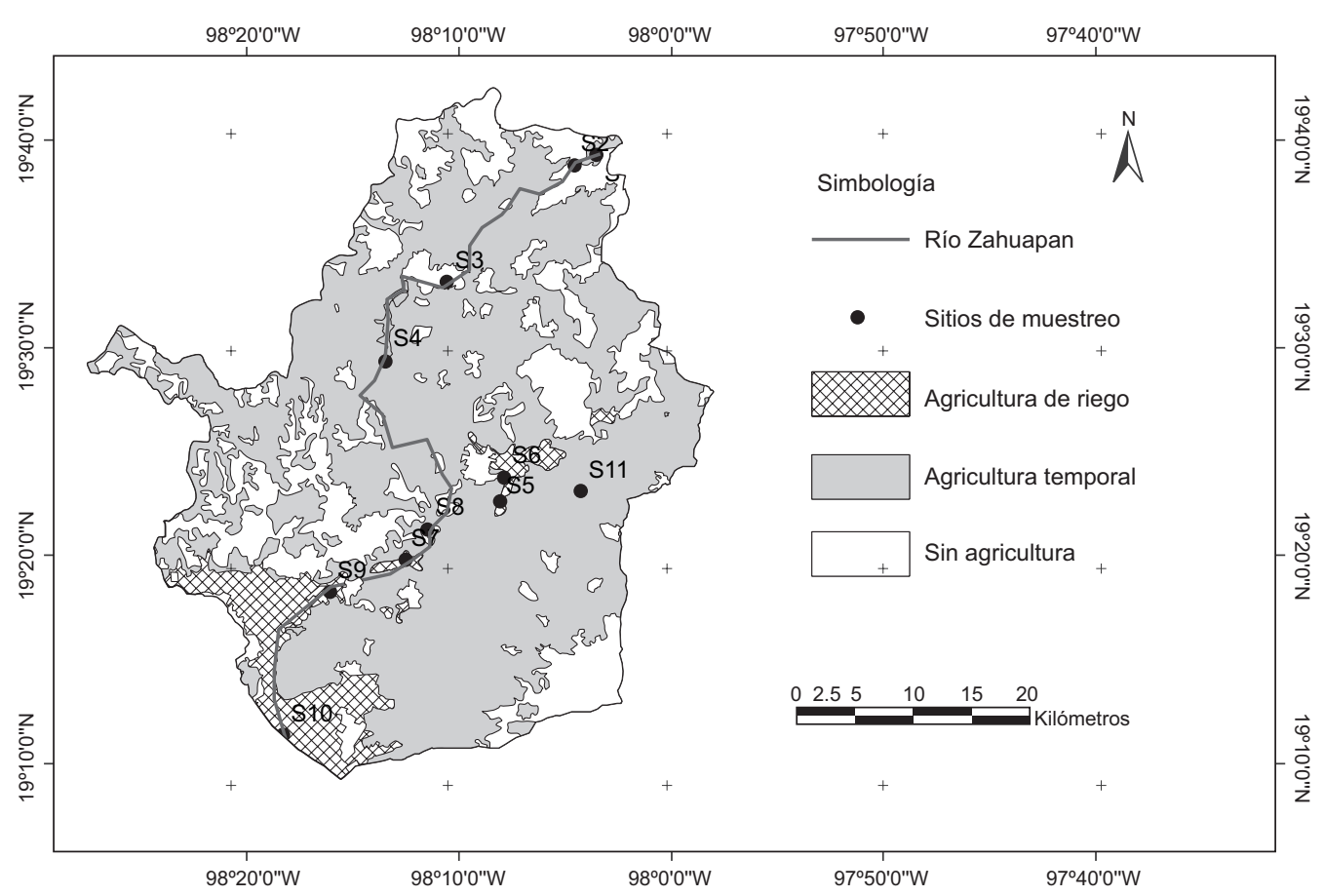

Fig. 3. Zonas agrícolas de la subcuenca del río Zahuapan

Los índices bióticos relevantes para evaluar la condición biótica del río fueron el IBH, el porcentaje de organismos tolerantes, el porcentaje de colectores, el porcentaje de depredadores, el número total de familias y el porcentaje de la familia dominante. El índice indicó que sólo los sitios de referencia presentaron una condición óptima. Los sitios S3 y S4, en cambio, una condición moderadamente impactada (Cuadro VII).
Estos sitios reciben un volumen menor de descargas de aguas residuales. Los sitios restantes mostraron una condición de hábitat pobre.

\section{Condición de hábitat}

El valor de los parámetros de condición de hábitat de los dos sitios de referencia fue mayor al de los sitios perturbados (Cuadro VIII). El análisis de

CUADRO VI. FAMILIAS DE MACROINVERTEBRADOS PRESENTES EN LOS SITIOS

\begin{tabular}{|c|c|c|c|c|c|c|c|c|c|c|c|c|}
\hline \multirow{2}{*}{ Familia } & \multirow{2}{*}{ Tolerancia* } & \multicolumn{11}{|c|}{ Sitios } \\
\hline & & $\mathrm{S} 1$ & $\mathrm{~S} 2$ & $\mathrm{~S} 3$ & S4 & S5 & S6 & S7 & S8 & S9 & S10 & S11 \\
\hline Elmidae & M & 14 & 11 & 0 & 0 & 0 & 0 & 0 & 0 & 0 & 0 & 0 \\
\hline Baetidae & M & 85 & 88 & 0 & 3 & 0 & 0 & 0 & 0 & 0 & 0 & 0 \\
\hline Corixidae & $\mathrm{A}$ & 1 & 2 & 0 & 3 & 0 & 0 & 0 & 0 & 0 & 0 & 0 \\
\hline Psychomyiidae & $\mathrm{B}$ & 59 & 47 & 0 & 0 & 0 & 0 & 0 & 0 & 0 & 0 & 0 \\
\hline Glossosomatidae & $\mathrm{B}$ & 52 & 65 & 0 & 0 & 0 & 0 & 0 & 0 & 0 & 0 & 0 \\
\hline Chironomidae & M & 17 & 15 & 0 & 5 & 0 & 0 & 0 & 0 & 0 & 0 & 0 \\
\hline Tipulidae & $\mathrm{B}$ & 6 & 9 & 0 & 0 & 0 & 0 & 0 & 0 & 0 & 0 & 0 \\
\hline Tubificidae & A & 0 & 0 & 31 & 0 & 0 & 0 & 0 & 0 & 250 & 0 & 0 \\
\hline Physidae & $\mathrm{A}$ & 0 & 0 & 0 & 52 & 0 & 0 & 0 & 0 & 0 & 0 & 0 \\
\hline Gammaridae & M & 0 & 0 & 214 & 0 & 0 & 0 & 0 & 0 & 0 & 0 & 0 \\
\hline Glossiphoniidae & A & 1 & 2 & 5 & 3 & 250 & 0 & 0 & 0 & 0 & 0 & 0 \\
\hline Daphniidae & A & 0 & 0 & 0 & 181 & 0 & 0 & 0 & 0 & 0 & 0 & 0 \\
\hline Perlidae & $\mathrm{B}$ & 15 & 17 & 0 & 3 & 0 & 0 & 0 & 0 & 0 & 0 & 0 \\
\hline Número total de organismos & & 250 & 250 & 250 & 250 & 250 & 0 & 0 & 0 & 250 & 0 & 0 \\
\hline Número total de familias & & 9 & 9 & 3 & 7 & 1 & 0 & 0 & 0 & 1 & 0 & 0 \\
\hline
\end{tabular}

${ }^{*} \mathrm{~A}=$ tolerancia alta, $\mathrm{M}=$ tolerancia moderada, $\mathrm{B}=$ tolerancia baja 
CUADRO VII. CONDICIÓN BIÓTICA DE LOS SITIOS

\begin{tabular}{lccl}
\hline Sitios & & $\begin{array}{r}\text { \% valor } \\
\text { del sitio }\end{array}$ & $\begin{array}{l}\text { Condición } \\
\text { biótica }\end{array}$ \\
\hline $\begin{array}{l}\text { Sitios de } \\
\text { referencia }\end{array}$ & S1 & 100.0 & Óptima \\
\hline Sitios perturbados & S3 & 25.0 & Óptima \\
& & & $\begin{array}{l}\text { Moderadamente } \\
\text { impactado }\end{array}$ \\
& S4 & 50.0 & $\begin{array}{l}\text { Moderadamente } \\
\text { impactado }\end{array}$ \\
& S5 & 0.0 & Pobre \\
& S6 & 0.0 & Pobre \\
& S7 & 0.0 & Pobre \\
& S8 & 0.0 & Pobre \\
S9 & 0.0 & Pobre \\
& S10 & 0.0 & Pobre \\
S11 & 0.0 & Pobre \\
\hline
\end{tabular}

conglomerados de los valores de condición de hábitat de los sitios, mostró una distinción clara entre los sitios de referencia y los sitios perturbados (Fig. 4). Dentro de los sitios perturbados, se observó la separación de tres grupos, lo que se asocia a la diferencia en el grado de perturbación del hábitat del río, causada por las actividades humanas.

El valor del índice de condición de hábitat indicó que a pesar de las diferencias en el grado de perturbación a lo largo del río, todos los sitios perturbados tienen una condición de hábitat pobre (Cuadro IX).

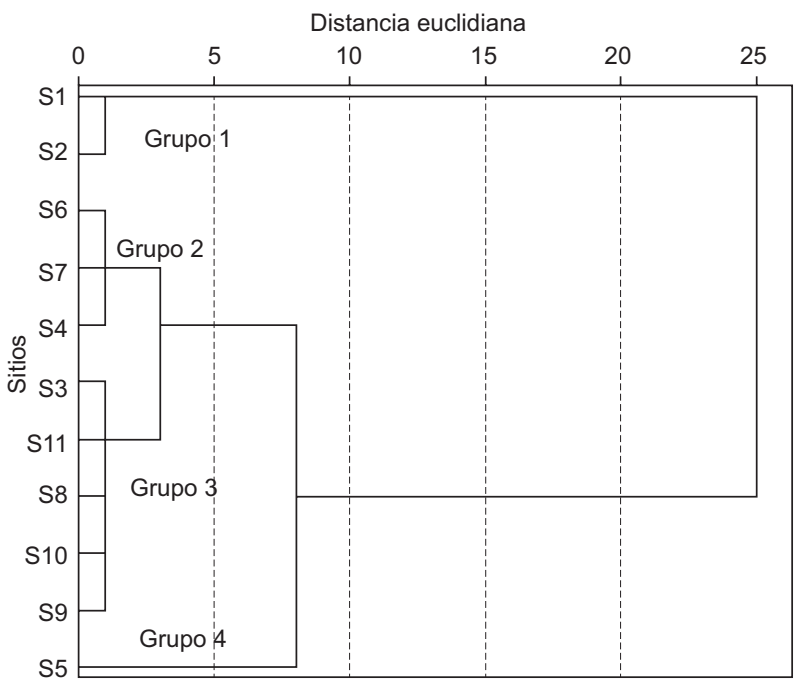

Fig. 4. Análisis de conglomerados del valor total de hábitat

\section{Condición de calidad del agua}

Los valores obtenidos de los nueve parámetros físicos y químicos tienden a ser mayores en los sitios perturbados que en los de referencia (Cuadro X), excepto el oxígeno disuelto, que es mayor en los sitios de referencia. Los valores de $\mathrm{pH}$ y temperatura son similares a los obtenidos por García-Nieto et al. (2011) y Muñoz-Nava et al. (2012) en sitios cercanos a la evaluación, excepto en los valores de temperatura registrados en la parte más alta de la subcuenca.

CUADRO VIII. VALORACIÓN DE LOS PARÁMETROS DE HÁBITAT

\begin{tabular}{|c|c|c|c|c|c|c|c|c|c|c|c|}
\hline \multirow[t]{2}{*}{ Parámetros } & \multicolumn{11}{|c|}{ Sitios } \\
\hline & S1 & $\mathrm{S} 2$ & S3 & S4 & S5 & S6 & S7 & S8 & S9 & $\mathrm{S} 10$ & S11 \\
\hline EBI & 10 & 10 & 3 & 3 & 6 & 3 & 3 & 2 & 2 & 2 & 2 \\
\hline EBD & 10 & 10 & 4 & 4 & 7 & 2 & 4 & 2 & 2 & 1 & 2 \\
\hline $\mathrm{AC}$ & 20 & 20 & 1 & 13 & 15 & 12 & 14 & 2 & 3 & 1 & 5 \\
\hline $\mathrm{EC}$ & 20 & 19 & 2 & 5 & 7 & 6 & 6 & 4 & 5 & 3 & 5 \\
\hline CCR & 20 & 20 & 1 & 7 & 8 & 6 & 7 & 3 & 8 & 7 & 2 \\
\hline RVP & 20 & 20 & 1 & 1 & 2 & 1 & 1 & 1 & 1 & 1 & 1 \\
\hline $\mathrm{SE} / \mathrm{CP}$ & 20 & 20 & 5 & 5 & 13 & 7 & 6 & 1 & 7 & 1 & 6 \\
\hline FR & 19 & 20 & 1 & 2 & 15 & 4 & 5 & 1 & 3 & 2 & 2 \\
\hline AZRBI & 10 & 10 & 2 & 2 & 8 & 4 & 3 & 1 & 1 & 0 & 1 \\
\hline AZRBD & 10 & 10 & 1 & 2 & 8 & 5 & 4 & 0 & 2 & 0 & 1 \\
\hline DS & 19 & 20 & 3 & 6 & 6 & 5 & 5 & 3 & 4 & 3 & 4 \\
\hline PVBD & 10 & 10 & 3 & 3 & 10 & 3 & 3 & 1 & 1 & 0 & 4 \\
\hline PVBI & 10 & 10 & 3 & 4 & 10 & 2 & 3 & 0 & 1 & 0 & 3 \\
\hline Total & 198 & 199 & 30 & 57 & 115 & 60 & 64 & 21 & 40 & 21 & 38 \\
\hline
\end{tabular}

$\mathrm{EBI}=$ estabilidad banco izquierdo, $\mathrm{EBD}=$ estabilidad banco derecho, $\mathrm{AC}=$ alteración del cauce, $\mathrm{EC}=$ estado del cauce, $\mathrm{CCR}$ = cubrimiento de cantos rodados por sedimentos finos, $\mathrm{RVP}=$ regímenes de velocidad y profundidad, $\mathrm{SE} / \mathrm{CP}=$ sustrato para la epifauna/cobertura disponible, $\mathrm{FR}=$ frecuencia de rápidos, $\mathrm{AZRBI}=$ ancho de la zona ribereña banco izquierdo, AZRBD = ancho de la zona ribereña banco derecho, DS $=$ depósito de sedimentos, $\mathrm{PVBD}=$ protección vegetal banco derecho, $\mathrm{PVBI}=$ protección vegetal banco izquierdo 
CUADRO IX. CONDICIÓN DE LOS SITIOS DE ACUERDO AL ESTADO DEL HÁBITAT

\begin{tabular}{llcc}
\hline Sitios & Factor de influencia & $\begin{array}{c}\text { Valor de } \\
\text { hábitat }\end{array}$ & Condición \\
\hline 1 & Bosque (sitio de referencia) & 0.990 & Óptima \\
2 & Bosque (sitio de referencia) & 0.995 & Óptima \\
3 & Actividades pecuarias & 0.150 & Pobre \\
4 & Actividades agrícolas & 0.285 & Pobre \\
5 & Uso urbano & 0.575 & Pobre \\
6 & Uso industrial - urbano & 0.300 & Pobre \\
7 & Uso industrial & 0.320 & Pobre \\
8 & Uso industrial - urbano & 0.105 & Pobre \\
9 & Uso urbano & 0.200 & Pobre \\
10 & Uso urbano & 0.105 & Pobre \\
11 & Uso agrícola & 0.190 & Pobre \\
\hline
\end{tabular}

En el presente estudio se obtuvieron valores de temperatura más bajos. Esto se atribuye a que en el día de la medición de temperatura en la zona, se presentó un descenso de la misma.

El índice de calidad del agua indicó que los sitios de referencia (S1 y S2) están en la categoría de excelente u óptima, asociada con una mínima perturbación por las actividades humanas (Cuadro XI). El sitio S4 presenta una condición favorable, debido a la presa Atlangatepec, ubicada aguas arriba del sitio, la cual recibe y retiene las descargas de aguas residuales. Los sitios S5 y S11 presentan una condición marginal, debido a un aumento de las descargas de las zonas urbanas e industriales sobre el río. Los sitios S3, S6, $\mathrm{S} 7, \mathrm{~S} 8, \mathrm{~S} 9$ y S10 presentan una condición pobre. En el sitio S3, ubicado en la entrada a la presa Atlangatepec, se concentran las aguas residuales del municipio
CUADRO XI. ÍNDICE DE CALIDAD DEL AGUA (ICA)

\begin{tabular}{lcc}
\hline Sitios & ICA & Categoría \\
\hline S1 & 100 & Excelente (Óptima) \\
S2 & 100 & Excelente (Óptima) \\
S3 & 28.28 & Pobre \\
S4 & 75.54 & Favorable \\
S5 & 52.07 & Marginal \\
S6 & 33.38 & Pobre \\
S7 & 24.90 & Pobre \\
S8 & 22.07 & Pobre \\
S9 & 25.59 & Pobre \\
S10 & 22.03 & Pobre \\
S11 & 46.04 & Marginal \\
\hline
\end{tabular}

de Tlaxco y su zona industrial. La condición de los otros sitios refleja la influencia permanente de grandes volúmenes de descargas de agua residual industrial y público-urbano.

\section{Asociación de los índices bióticos de condición biótica, condición de hábitat y calidad del agua}

El análisis de correlación de Spearman mostró que el número de familias del orden Trichoptera, el porcentaje de EPT, el porcentaje de Ephemeroptera, el porcentaje de raspadores y el porcentaje de fragmentadores se relacionan positivamente con la puntuación total de hábitat (Fig. 5). El valor de estos índices bióticos disminuye si el hábitat se encuentra perturbado (Barbour et al. 1999, Tetra Tech 2000). Estos resultados son similares a los obtenidos por Ríos y Bailey (2006), Sponseller et al. (2001) y Boosoong et al. (2009), quienes encontraron para sitios forestales y una mínima perturbación, valores más

CUADRO X. PARÁMETROS FÍSICOS Y QUÍMICOS DE LOS SITIOS

\begin{tabular}{|c|c|c|c|c|c|c|c|c|c|c|c|c|}
\hline \multirow[t]{2}{*}{ Parámetros } & \multirow[t]{2}{*}{ VR } & \multicolumn{11}{|c|}{ Sitios } \\
\hline & & $\mathrm{S} 1$ & $\mathrm{~S} 2$ & S3 & S4 & S5 & S6 & S7 & S8 & S9 & $\mathrm{S} 10$ & S11 \\
\hline Temperatura $\left({ }^{\circ} \mathrm{C}\right)$ & $<40^{\circ} \mathrm{C}$ (a) & 1.40 & 1.50 & 14.10 & 5.50 & 11.0 & 11.20 & 8.60 & 11.30 & 17.50 & 16.70 & 13.40 \\
\hline Oxígeno disuelto & & & & & & & & & & & & \\
\hline $\begin{array}{l}(\mathrm{mg} / \mathrm{l}) \\
\text { Conductividad }(\mu \mathrm{s} / \mathrm{cm})\end{array}$ & 5.5 (b) & $\begin{array}{c}6.96 \\
124.4\end{array}$ & $\begin{array}{c}6.94 \\
129.2\end{array}$ & $\begin{array}{l}1.70 \\
1076\end{array}$ & $\begin{array}{l}3.22 \\
923\end{array}$ & $\begin{array}{l}3.29 \\
413\end{array}$ & $\begin{array}{l}2.22 \\
2220\end{array}$ & $\begin{array}{l}2.54 \\
2210\end{array}$ & $\begin{array}{l}2.25 \\
2530\end{array}$ & $\begin{array}{c}2.6 \\
2270\end{array}$ & $\begin{array}{l}2.38 \\
5130\end{array}$ & $\begin{array}{l}3.28 \\
752\end{array}$ \\
\hline Total de sólidos & & & & & & & & & & & & \\
\hline disueltos (mg/l) & $<1000$ (c) & 59 & 60.70 & 525 & 43.60 & 197.80 & 1115 & 1745 & 1491 & 1241 & 5890 & 363 \\
\hline Salinidad (mg/l) & & 0 & 0 & 0.50 & 0.30 & 0.20 & 1.10 & 1.80 & 1.50 & 1.40 & 1.50 & 0.30 \\
\hline $\mathrm{pH}$ & $6.5-9(b)$ & 7.80 & 7.80 & 8.00 & 7.10 & 7.50 & 8.10 & 8.51 & 8.40 & 8.24 & 8.20 & 7.80 \\
\hline Amonio (mg/l) & 19 (b) & 0 & 0 & 20 & 2 & 20 & 50 & 50 & 102 & 45 & 100 & 9 \\
\hline Amoniaco (mg/l) & & 0 & 0 & 0.490 & 0.002 & 0.126 & 0.985 & 2.580 & 2.000 & 1.489 & 3.080 & 0.207 \\
\hline $\mathrm{DBO}_{5}(\mathrm{mg} / \mathrm{l})$ & 60 (a) & 0 & 0 & 170 & 90 & 100 & 300 & 320 & 450 & 270 & 350 & 130 \\
\hline Fósforo total (mg/l) & 10 (a) & 0 & 0 & 50 & 3 & 3 & 72 & 70 & 100 & 55 & 98 & 25 \\
\hline
\end{tabular}

VR = valor de referencia, (a) VR de la NOM-001-SEMARNAT-1996, (b) VR de la Norma Canadiense para la Protección de Vida Acuática (CCME 2007), (c) VR del estado de Iowa (IDNR 2009) 


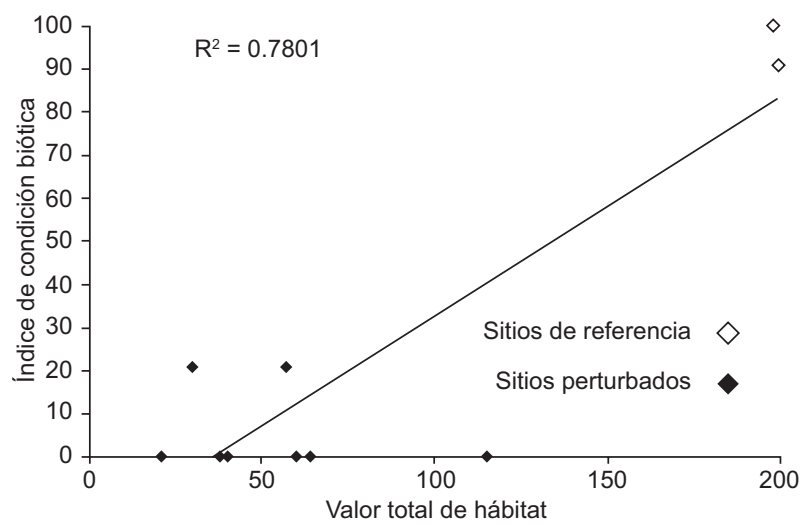

Fig. 5. Relación entre el valor total de hábitat y el índice de condición biótica

altos de porcentaje de EPT, porcentaje de Ephemeroptera y número de familias del orden Trichoptera.

Los parámetros de calidad del agua que mostraron mayor asociación con los índices de condición biótica del río fueron la demanda biológica de oxígeno para un periodo de cinco días $\left(\mathrm{DBO}_{5}\right)$, el fósforo total y la temperatura $(8,7$ y 6 correlaciones significativas, respectivamente). Las correlaciones significativas fueron con el número total de familias, número de familias de EPT, número de familias del orden Trichoptera, porcentaje de Ephemeroptera y porcentaje de raspadores. Todos estos índices bióticos son indicadoras de buena calidad del sitio.

En términos generales, se confirmó que los índices bióticos de condición biótica que se reportan como indicadores de una mejor calidad del agua, se asociaron en mayor proporción con los parámetros físicos y químicos de calidad del agua. Estos resultados indican que tanto la condición del hábitat como los diferentes tipos de descargas de aguas residuales inciden significativamente en la condición biótica del río Zahuapan.

\section{Condición ecológica global del río Zahuapan}

La evaluación global de la condición del río indicó que todos los sitios perturbados tienen un estado pobre (la condición más baja o más impactada), a excepción del sitio S4 (Cuadro XII), localizado aguas abajo de la presa Atlangatepec. Esto se debe a que no recibe grandes volúmenes de aguas residuales y a que la presa retiene contaminantes de las descargas vertidas aguas arriba. Se considera que, en términos generales, hubo concordancia en las tres evaluaciones de la condición del río. Aunque la evaluación de calidad de agua (parámetros físicos y químicos), mostró una ligera diferenciación entre los sitios, seguida de la evaluación biótica.

\section{CONCLUSIÓN}

El enfoque multimétrico, basado en el protocolo de evaluación biótica en ecosistemas acuáticos, tomando como bioindicadores a los macroinvertebrados, permitió evaluar la condición ecológica de los sitios donde hay un marcado impacto de las actividades humanas. Se logró diferenciar claramente los sitios tomados como referencia, con mínima perturbación de los sitios perturbados por actividades antrópicas. Comparadas con la evaluación global (que integra la condición de hábitat, biótica y de calidad de agua), las evaluaciones de hábitat y biótica asignaron un grado de condición diferente a un sitio, mientras que la evaluación biótica difirió con la evaluación global en dos sitios. En general, todas las evaluaciones indican que las actividades pecuarias, urbanas e industriales

CUADRO XII. CONDICIÓN DE INTEGRIDAD ECOLÓGICA GLOBAL

\begin{tabular}{llllc}
\hline \multirow{2}{*}{ Sitios } & \multicolumn{3}{c}{ Condición } & Condición general \\
\cline { 2 - 4 } & Hábitat & Biótica & Calidad de agua & \\
\hline S1 & Óptima & Óptima & Excelente (Óptima) & Óptima \\
S2 & Óptima & Óptima & Excelente (Óptima) & Óptima \\
S3 & Pobre & Moderadamente impactada & Pobre & Pobre \\
S4 & Pobre & Moderadamente impactada & Favorable & Moderadamente impactada \\
S5 & Pobre & Pobre & Marginal & Pobre \\
S6 & Pobre & Pobre & Pobre & Pobre \\
S7 & Pobre & Pobre & Pobre & Pobre \\
S8 & Pobre & Pobre & Pobre & Pobre \\
S9 & Pobre & Pobre & Pobre & Pobre \\
S10 & Pobre & Pobre & Pobre & Pobre \\
S11 & Pobre & Pobre & Marginal & Pobre \\
\hline
\end{tabular}


tienen un impacto negativo en la condición ecológica del Río Zahuapan. La condición ecológica global de este río, a excepción de las zonas forestales donde la perturbación por las actividades humanas es mínima, se ubica en la categoría de pobre, que corresponde a la peor condición ecológica de los métodos utilizados en esta evaluación.

Los resultados mostraron que las variables de hábitat, bióticas y de calidad del agua están relacionadas entre sí, por lo que la restauración de la condición ecológica del río debe considerar a las tres variables. Esto requiere la adopción de un enfoque de manejo integral de cuencas. Asimismo, las actividades pecuarias y las descargas de aguas residuales urbanas e industriales son los principales factores de deterioro de la condición del río Zahuapan. La influencia de las actividades agrícolas es más localizada y de menor impacto que las derivadas de los asentamientos humanos y desarrollos industriales.

\section{REFERENCIAS}

Barbour M.T., Gerritsen J., Snyder B.D. y Stribling J.B. (1999). Protocols for use in streams and weadable rivers: periphyton, benthic macroinvertebrates and fish. 2a ed. U.S. Environmental Protection Agency, Office of Water. Washington, EUA, 339 pp.

Boonsoong B., Sangpradub N. y Barbour M. T. (2009). Development of rapid bioassessment approaches using benthic macroinvertebrates for Thai streams. Environ. Monit. Assess. 155, 129-147.

DOI: $10.1007 / \mathrm{s} 10661-008-0423-2$.

Bouchard R. W. Jr. (2004). Guide to aquatic macroinvertebrates of the Upper Midwest Resources Center. University of Minnesota. St. Paul, EUA, 208 pp.

CCME (2001). Canadian water quality guidelines for the protection of aquatic life: CCME Water Quality Index 1.0, user's manual. Canadian Council of Ministers of the Environment. Winnipeg, Canada, 5 pp.

CCME (2007). Canadian water quality guidelines for freshwater aquatic life: summary table. Canadian Council of Ministers of the Environment. Winnipeg, Canada, 9 pp.

CONAGUA (2012). Localizador de aprovechamientos. [en línea]. https://www.gob.mx/conagua/acciones-yprogramas/localizador-de-aprovechamientos-locrepda 07/01/2017.

Correa-Araneda F., Rivera R., Urrutia J., De los Ríos P., Contreras A. y Encina-Montoya F. (2010). Efectos de una zona urbana sobre la comunidad de macroinvertebrados bentónicos de un ecosistema fluvial del sur de Chile. Limnetica 29, 183-194.
Frissell C.A., Liss W.J., Warren C.E. y Hurley M.D. (1986). A hierarchical framework for stream habitat classification: viewing streams in a watershed context. Environm. Manage. 2, 199-214.

DOI: $10.1007 / \mathrm{BF} 01867358$.

Gamboa M., Reyes R. y Arrivillaga J. (2008). Macroinvertebrados bentónicos como bioindicadores de salud ambiental. Boletín de Malariología y Salud Ambiental 2, 109-120.

García-Nieto E., Carrizales-Yañez L., Juárez-Santacruz L., García-Gallegos E., Hernández-Acosta E., BrionesCorona E. y Vázquez-Cuecuecha O. G. (2011). Plomo y arsénico en la subcuenca del Alto Atoyac en Tlaxcala, México. Rev. Chapingo Ser. Cie. 1, 7-17.

DOI: $10.5154 /$ r.rchscfa.2010.06.040.

Godínez-Domínguez E. y González-Sansón G. (1999). La diversidad de macroinvertebrados de fondos blandos de la plataforma continental de Jalisco y Colima, México. Cienc. Mar. 4, 609-627.

Gutiérrez-Fonseca P. E. (2010). Guía ilustrada para el estudio ecológico y taxonómico de los insectos acuáticos del Orden Coleóptera en El Salvador. Editorial Universitaria UES, San Salvador, El Salvador, 64 pp.

HACH (2003). Water analysis handbook. 4th ed. Hach Company. Loveland, EUA, 1261 pp.

Harrison T.D. y Whitfield A.K. (2006). Application of a multimetric fish index to assess the environmental condition of South African estuaries. Estuar. Coast. 6, 1108-1120.

Herbst D. B. y Silldorff E. L. (2009). Development of a benthic macroinvertebrate index of biological integrity (IBI) for stream assessments in the eastern Sierra Nevada of California. Reporte Final. California Environmental Protection Agency. Los Angeles, EUA, 89 pp.

IDNR (2009). Water quality standards review: Chloride, sulfate and total dissolved solids. Iowa Department of Natural Resources. Des Moines, EUA, 79 pp.

INEGI (2010). Censo General de Población y Vivienda, México [en línea]. http://www.inegi.org.mx/est/contenidos/proyectos/ccpv/cpv2010/] 10/01/2017.

Karr J.R. (2006). Seven foundations of biological monitoring and assessment. Biologia Ambientale 2, 7-18.

Li L., Zheng B. y Liu L. (2010). Biomonitoring and bioindicators used for river ecosystems: definitions, approaches and trends. Procedia Environmental Sciences 2, 1510-1524. DOI: 10.1016/j.proenv.2010.10.164

Lyons J., Navarro-Pérez S., Cochran P.A., Santana E. y Guzmán-Arroyo M. (1995). Index of biotic integrity based on fish assemblages for the conservation of streams and rivers in west-central Mexico. Conserv. Biol. 3, 569-584.

DOI: $10.1046 / \mathrm{j} .1523-1739.1995 .09030569 . x$

Martins R.T., Stephan N.N.C. y Alves R.G. (2008). Tubificidae (Annelida: Oligochaeta) as an indicator of 
water quality in an urban stream in southeast Brazil. Acta Limnol. Bras. 20, 221-226.

Moreno P. y. Callisto M. (2006). Benthic macroinvertebrates in the watershed of an urban reservoir in southeastern Brazil. Hydrobiología 560, 311-321.

Muñoz-Nava H., Suarez-Sánchez J., Vera-Reyes A., Orozco-Flores S., Batlle-Sales J., Ortiz-Zamora A. J. y Mendiola-Argüelles J. (2012). Demanda Bioquímica de Oxígeno y población en la subcuenca del río Zahuapan en Tlaxcala, México. Rev. Int. Contam. Ambie. 28, 27-38.

Oliveira S.V. y Cortes R.M.V. (2006). Environmental indicators of ecological integrity and their development for running waters in northern Portugal. Limnetica 1-2, 479-498.

Oscoz E. J. (2009). Guía de campo de macroinvertebrados de la cuenca del Ebro. Confederación Hidrográfica del Ebro. Zaragoza, España, 126 pp.

Plafkin J. L., Barbour M.T., Porter K. D., Gross S. K. y Hughes R. M. (1989). Rapid bioassessment protocols for use in streams and rivers: benthic macroinvertebrates and fish. EPA 440/4-89/001. Office of Water, U.S. Environmental Protection Agency Washington, D.C., EUA, 179 pp.

Richards K. y Horn R. (2005). Guidelines for the assessment of the hydromorphological status of rivers as part of the requirements of the Water Framework Directive: I. Principles. Environment and Heritage Service. Belfast, Northern Ireland, 79 pp.
Rios S. L. y Bailey R. C. (2006). Relationship between riparian vegetation and stream benthic communities at three spatial scales. Hydrobiologia 553, 153-160. DOI: $10.1007 / \mathrm{s} 10750-005-0868-\mathrm{Z}$

Schallenberg M., Kelly D., Clapcott J Death., R., MacNeil C., Young R., Sorrell B. y Scarsbrook M. (2011). Approaches to assessing ecological integrity of New Zealand freshwaters. New Zealand Department of Conservation. Science for Conservation 307. Wellington, Nueva Zelandia, 84 pp.

Serrano C. L. y Zepeda A. (2010). Guía ilustrada para el estudio ecológico y taxonómico de los insectos acuáticos inmaduros del orden Ephemeroptera en El Salvador. Editorial Universitaria UES. San Salvador, El Salvador, 29 pp.

Sponseller R. A., Benfield E. F. y Valett H. M. (2001). Relationships between land use, spatial scale and stream macroinvertebrate communities. Freshwater Biol. 46, 1409-1424. DOI: 10.1046/j.1365-2427.2001.00758.x

Tetra Tech (2000). A stream condition index for West Virginia wadeable streams. Owings Mills, MD, EUA, 80 pp.

VANR (2004). Vermont stream geomorphic assessment phase 2 handbook. Rapid stream assessment. Vermont Agency of Natural Resources. Montpelier, EUA, 83 pp.

Weigel B.M., Henne L.J. y Martínez-Rivera L.M. (2002). Macroinvertebrate-based index of biotic integrity for protection of streams in west-central Mexico. J. N. Am. Benthol. Soc. 4, 686-700. DOI: 10.2307/1468439 